B. Jefferies

Nagoya Math. J.

Vol. 115 (1989), 47-62

\title{
INTEGRATION WITH RESPECT TO THE PRODUCT OF A MEASURE AND A CLOSABLE SET FUNCTION
}

\author{
BRIAN JEFFERIES*
}

\section{Introduction}

The notion of a set function being closable with respect to a family of measures was introduced in [1] and applied there to the integration of Feynman-Kac functionals. In [2], it is shown how the interchange of integrals for the product of an operator valued measure and a scalar measure can be used to solve operator equations associated with the perturbation of semigroups. The purpose of this article is to develop the analogous machinery for closable set functions with the view of applying it to Schrödinger's equation.

It turns out that the behaviour of closable set functions with respect to interchanging integrals bears a resemblance to that of vector valued measures; it should not be surprising, because many examples of scalar valued closable set functions can be constructed from operator valued measures.

The main feature of product integrals with respect to both of these classes of set functions, is that as long as we integrate with respect to the scalar measure first, and then with respect to the set function, then the result is equal to the integral with respect to the product of the two. However, in reversing that order of integration, the integral with respect to the vector valued measure may not exist, almost everywhere, with values in the space in question.

An outline of integration with respect to closable set functions introduced in [1] is given in Section 2. The main result, Theorem 3.5, on integration with respect to the product of a closable set function and a measure is given in Section 3. A partial result on the previously mentioned interchange of the orders of integration requires a stronger form

Received November 2, 1987.

* Research supported by a Queen Elizabeth II Fellowship. 
of closability for set functions with values in a nuclear Fréchet space. The conditions exclude scalar valued closable set functions which are not themselves $\sigma$-additive, but even in that case, the result gives new information; see Example 3.6.

The techniques are applied in Section 4 to represent the solution of Schrödinger's equation with a bounded potential in terms of the integral with respect to a closable set function. The representation is derived in [1] via the Trotter product formula. The present derivation is used in Corollary 4.3 to characterize solutions of Schrödinger's equation obtained by path integration in terms of the potential $V \in L_{\mathrm{loc}}^{2}\left(\mathbb{R}^{d}\right)$.

The present paper has a close relationship with [3], where the notion of the closability of a set function with respect to a measure is also inherent. The point of departure in Section 4 is that analytic continuation of the set functions is used to obtain the family of measures with respect to the closure is taken, thereby avoiding the use of Banach limits introduced in Section 2.D of [3]. The relationship between the dynamical semigroup, and the corresponding Hamiltonian operator is more transparent in the present formulation.

\section{$\S 2$. Closable set functions}

In this section, the terminology and notation from [1] is given by way of introduction to those ideas used in applications to Feynman integrals. The following setup is used to integrate with respect to a family $\Lambda$ of additive vector valued set functions, relative to another family $\Gamma$ of non-negative measures, with respect to which $\Lambda$ is closable.

There is a semi-algebra $\subseteq$ of subsets of the non-empty set $\Omega$, and an increasing collection $\left\langle\widetilde{S}_{\zeta}\right\rangle_{\zeta \in Z}$ of non-empty sub-semi-algebras of $\widetilde{\varsigma}$ such that $\cup_{\zeta \in Z} \widetilde{\Im}_{\zeta}=$ S. A semi-algebra $\widetilde{S}$ of subsets of a set $\Omega$ is a collection of sets containing $\Omega$ and $\varnothing$, closed under finite intersections, and with the property that if $A \in \mathfrak{S}, B \in \mathbb{S}$, then $A \backslash B$ is equal to the union of finite family of pairwise disjoint sets $U_{j}, j=0,1, \ldots, k$ belonging to $\mathfrak{S}$, which are numbered so that if $U_{0}=A \cap B$, then the union $\bigcup_{j=0}^{i} U_{j}$ belongs to $\mathfrak{S}$ for every $i=0,1, \cdots, k$. A characteristic example is the collection of products of half-open intervals $\left[a_{i}, b_{i}\right), i=1, \cdots, d$ in $\mathbb{R}^{d}$.

For a given locally convex space $E, A=\left\{\Lambda_{\xi}: \xi \in W_{1}\right\}$ is a collection of additive set functions $m: \subseteq \rightarrow E$ such that for each $\xi \in W_{1}$, the set $\Lambda_{\xi}$ is a bounded subset of the space $\mathrm{ba}_{\zeta \in Z}\left(\Im_{\zeta}, E\right)$. In this notation, $\underset{\leftarrow}{\mathrm{ba}_{\zeta \in Z}}\left(\widetilde{S}_{\zeta}, E\right)$ 


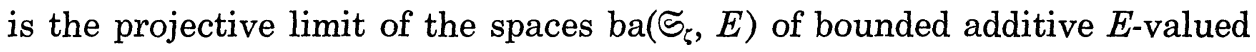
set functions on the semi-algebras $\widetilde{S}_{\zeta}, \zeta \in Z$, equipped with the uniform norm. Thus, for each $\zeta \in Z$, the set of restrictions of the set functions in $\Lambda_{\xi}$ to sets in the semi-algebra $\widetilde{\varsigma}_{\xi}$ is uniformly bounded. Note that no set function need be bounded on the algebra generated by $\mathfrak{S}_{\zeta}$.

The collection $\Gamma=\left\{\Gamma_{\xi}: \xi \in W_{0}\right\}$ consists of families of measures $\mu: \sigma(\mathfrak{S}) \rightarrow[0, \infty)$ defined on the $\sigma$-algebra $\sigma(\mathfrak{S})$ generated by $\mathfrak{S}$, such that for each $\xi \in W_{0}$, the set $\Gamma_{\xi}$ is bounded in the variation norm (that is, $\sup \left\{\mu(\Omega): \mu \in \Gamma_{\xi}\right\}<\infty$ ), and uniformly countably additive. Additionally, it is assumed that the simple functions are dense in $L^{1}(\Gamma)$-the space of equivalence classes of functions $f: \Omega \rightarrow \mathbb{R}$ such that for each $\xi \in W_{0}$, $p_{\xi}(f)=\sup \left\{\mu(f) ; \mu \in \Gamma_{\xi}\right\}<\infty$. The space $L^{1}(\Gamma)$ is equipped with the family $\left\{p_{\xi}: \xi \in W_{0}\right\}$ of seminorms.

Now the notion that $\Lambda$ should be closable with respect to $\Gamma$ can be expressed as follows. Let $\mathscr{P}$ be a fundamental collection of seminorms

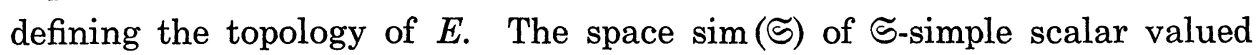
functions can be endowed with two distinct topologies.

First, there is the topology $\tau_{\Gamma}$ induced by the locally convex topology of $L^{1}(\Gamma)$, and then there is another topology $\tau_{A}$ induced by the family $\Lambda$, defined by the collection of seminorms $\left\{p_{\zeta \xi}: \zeta \in Z, \xi \in W_{1}, p \in \mathscr{P}\right\}$. For each $p \in \mathscr{P}, \zeta \in Z, \xi \in W_{1}$, and $s \in \operatorname{sim}(\widetilde{S})$.

$$
p_{\zeta \xi}(s)=\sup \left\{p(\operatorname{sm}(A)): A \in \Im_{\zeta}, m \in \Lambda_{\xi}\right\} \text {. }
$$

The topologies $\tau_{\Lambda}$ and $\tau_{\Gamma}$ can be made to be Hausdorff by taking the appropriate quotient spaces $\operatorname{sim}(\widetilde{S})_{\Lambda}, \operatorname{sim}(\widetilde{S})_{\Gamma}$ of $\operatorname{sim}(\widetilde{S})$, so that $\operatorname{sim}(\widetilde{S})$ becomes a locally convex Hausdorff space under both of the topologies.

Provided that the space $L^{1}(\Gamma)$ is complete, and the families $\Gamma$ and $\Lambda$ are compatible in the sense that the identity map $I$ on $\operatorname{sim}(\Im)$ factors through $\operatorname{sim}(\widetilde{S})_{\Gamma}$ and $\operatorname{sim}(\mathbb{S})_{A}$ into a map $I_{\Gamma A}$, then $\Lambda$ is said to be $\Gamma$ closable if the identity map on $\operatorname{sim}(\widetilde{S})_{A}$ is a closable linear map from $L^{1}(\Gamma)$ into the completion of $\left\langle\operatorname{sim}(\widetilde{S})_{\Lambda}, \tau_{A}\right\rangle$; that is, the closure of its graph in the product topology is the graph of an operator.

The integration map $s \rightarrow s m, s \in \operatorname{sim}(\widetilde{S})$, is clearly continuous for $\tau_{A}$ into $\mathrm{ba}_{\zeta \in Z}\left(\widetilde{S}_{\zeta}, E\right)$, so a function $f: \Omega \rightarrow \mathbb{C}$ is said to be $\Gamma$ - $\Lambda$-integrable if $f$ belongs to the domain $\mathfrak{D}\left(\bar{I}_{\Gamma A}\right)$ of the closure $\bar{I}_{\Gamma \Lambda}$ of the map $I_{\Gamma \Lambda}$, and for every $m \in \cup \Lambda, f m \in \mathrm{ba}_{\zeta \in Z}\left(\widetilde{S}_{\zeta}, E\right)$. Here, the indefinite integral fm is defined by continuity. If $E$ is complete, then this last condition follows auto- 
matically from $f \in \mathfrak{D}\left(\bar{I}_{\Gamma A}\right)$. A convergence theorem can be read off from the definition [1, Theorem 2.5].

That a collection of set functions should be closable with respect to a family of measures may be viewed as a minimal requirement of a discontinuous integration process. The setup is devised to deal wtih the Feynman integral as follows.

Let $K$ be the positive imaginary axis $\{a \mathrm{i}: a>0\}$, and let $D$ be the upper half plane $\{z \in \mathbb{C}: \operatorname{Im} z \geq 0, z \neq 0\}$, excluding the origin.

Let $H_{0}=-\frac{1}{2} \Delta$ be the free Hamiltonian operator of a quantum system in $\mathbb{R}^{d}$; the operator $\Delta$ is the self-adjoint extension on $L^{2}\left(\mathbb{R}^{d}\right)$ of the Laplacian operator $\frac{\partial^{2}}{\partial x_{1}^{2}}+\cdots+\frac{\partial^{2}}{\partial x_{d}^{2}}$ defined on all smooth functions of compact support on $\mathbb{R}^{d}$. Then for each $z \in D$, the operator $S^{z}(t)=e^{-i H_{0} t / z}$ is defined by the operational calculus for self-adjoint operators on $L^{2}\left(\mathbb{R}^{d}\right)$.

The set of all continuous functions or paths $\omega:[0, \infty) \rightarrow \mathbb{R}^{d}$ is denoted by $\Omega$, and $X_{t}(\omega)=\omega(t), t \geq 0$ define random variables on $\Omega$. For each $t>0$, $\varsigma_{t}$ denotes the semi-algebra of all subsets of $\Omega$ of the form $\left\{X_{t_{1}} \in B_{1}\right.$, $\left.X_{t_{2}} \in B_{2}, \cdots, X_{t_{k}} \in B_{k}\right\}$ for times $0<t_{1}<t_{2}<\cdots<t_{k} \leq t$ and Borel sets $B_{1}, B_{2}, \cdots, B_{k}$ in $\mathbb{R}^{d}, k=1,2, \cdots$. Then obviously $\widetilde{S}_{t}$ is the union $\cup_{\xi \in Z} \Im_{\xi}$ of the semi-algebras $\widetilde{S}_{\xi}$ obtained from the collection of sets of the above type, when the times $\zeta=\left\{t_{1}, \cdots, t_{k}\right\}, 0<t_{1}<t_{2}<\cdots<t_{k} \leq t$ are fixed, and the Borel sets $B_{1}, B_{2}, \cdots, B_{k}$ vary.

For each $t>0$, and $z \in D$, set

$$
\begin{aligned}
M_{t}^{z}\left(\left\{X_{t_{1}}\right.\right. & \left.\left.\in B_{1}, \cdots, X_{t_{k}} \in B_{k}\right\}\right) \\
& =S^{z}\left(t-t_{k}\right) B_{k} S^{z}\left(t_{k}-t_{k-1}\right) B_{k-1} \cdots B_{2} S^{z}\left(t_{2}-t_{1}\right) B_{1} S^{z}\left(t_{1}\right),
\end{aligned}
$$

for all $0<t_{1}<t_{2}<\cdots<t_{k} \leq t, B_{1}, B_{2}, \cdots, B_{k}$ Borel sets in $\mathbb{R}^{d}$, and $k=1,2, \cdots$. Here we have identified a Borel set in $\mathbb{R}^{a}$ with the operator on $L^{2}\left(\mathbb{R}^{d}\right)$ of multiplication by the set's characteristic function.

Then $M_{t}^{z}: \Im_{t} \rightarrow \mathfrak{L}\left(L^{2}\left(\mathbb{R}^{d}\right)\right)$ is an additive operator valued set function. For each $z \in K, M_{t}^{z}$ is the restriction to $\Im_{t}$ of a unique operator valued measure, also denoted by $M_{t}^{z}$, on the $\sigma$-algebra $\sigma\left(\widetilde{S}_{t}\right)$ generated by $\widetilde{S}_{t}$. This follows by representing $M_{t}^{z}$ in terms of the Wiener process [1, p. 389].

Let $\Gamma_{t}$ be the collection,

$$
\begin{gathered}
\left\{\Gamma_{t}^{a, \phi}: a>0, \phi \in L^{2}\left(\mathbb{R}^{d}\right),\|\phi\|_{2} \leq 1\right\}, \\
\Gamma_{t}^{a, \phi}=\left\{\left|\left(M_{t}^{z} \phi, \psi\right)\right|: \sigma\left(\mathbb{S}_{t}\right) \rightarrow[0, \infty): z=a \mathfrak{i}, \psi \in L^{2}\left(\mathbb{R}^{d}\right),\|\psi\|_{2} \leq 1\right\} .
\end{gathered}
$$


Here the variation of a signed measure $m$ is denoted by $|m|$.

The locally convex space $E$ is taken to be the space $H_{0}(D)$ of functions analytic in the interior $D^{\circ}$ of $D$, and continuous on $D$, endowed with the topology of uniform convergence on compact subsets of $D^{\circ}$. The space $H_{c}(D)$ is not complete. The $E$-valued set functions associated with the quantum process are defined as follows.

For each $\phi, \psi \in L^{2}\left(\mathbb{R}^{d}\right), t>0,\left(M_{t}^{D} \phi, \psi\right)$ represents the $H_{c}(D)$-valued set function on $\widetilde{S}_{t}$ defined by $\left(M_{t}^{D} \phi, \psi\right)(A)(z)=\left(M_{t}^{z} \phi, \psi\right)(A)$ for all $z \in D$, and $A \in \widetilde{S}_{t}$. Now define the collection $\Lambda_{t}$ to be the collection

$$
\begin{gathered}
\left\{\Lambda_{t}^{\phi}: \phi \in L^{2}\left(\mathbb{R}^{d}\right),\|\phi\|_{2} \leq 1\right\}, \\
\Lambda_{t}^{\phi}=\left\{\left(M_{t}^{D} \phi, \psi\right): \widetilde{S}_{t} \rightarrow H_{c}(D): \psi \in L^{2}\left(\mathbb{R}^{d}\right),\|\psi\|_{2} \leq 1\right\} .
\end{gathered}
$$

Then $\Lambda_{t}$ is $\Gamma_{t}$-closable [1, Theorem 3.4]. A $\Gamma_{t}$ - $\Lambda_{t}$-integrable function is said to be $M_{t}^{K}-M_{t}^{D}$-integrable. A $\Gamma_{t}$-integrable function is said to be $M_{t}^{K}$-integrable. For each $M_{t}^{K}-M_{t}^{D}$-integrable function $f$, the additive operator-valued set functions $f M_{t}^{z}: \Im_{t} \rightarrow \mathfrak{L}\left(L^{2}\left(\mathbb{R}^{d}\right)\right), z \in D$, can be read off from the definitions in the obvious way [1, Theorem 3.5]. For $z \in K$, they correspond to the usual integrals with respect to an operator-valued measure [4], and for each $t>0, A \in \mathfrak{S}_{t}, f M_{t}^{*}(A): D \rightarrow \mathfrak{L}\left(L^{2}\left(\mathbb{R}^{d}\right)\right)$ is continuous in the weak operator topology on all of $D$, and analytic in the interior of $D$.

The same construction is valid if the set $D$ is replaced by any set $F$ in the upper half-plane, whose interior contains the positive imaginary axis. In this case, the set function $M_{t}^{D}$ is replaced by the set function $M_{t}^{F}$ in the above expressions.

\section{§ 3. Product integration}

In this section, $\Lambda$ is taken to be a collection of $E$-valued set functions on the semi-algebra $\widetilde{S}$, which is closable with respect to the collection $\Gamma$ of measures on $\sigma(\widetilde{S})$, as outlined in Section 2.

Let $\nu: \mathfrak{I} \rightarrow[0, \infty[$ be a measure defined on a $\sigma$-algebra $\mathfrak{I}$ of subsets of a set $\Sigma$. For each $\mu \in \cup \Gamma, \mu \otimes \nu$ denotes the product of the measures $\mu$ and $\nu$ defined on the $\sigma$-algebra $\sigma(\widetilde{S}) \otimes \mathfrak{I}$ generated by rectangles in $\Omega \times \Sigma$.

For each $m \in \cup \Lambda$, define $m \times \nu: \mathbb{S} \times \mathfrak{T} \rightarrow E$ by

$$
m \times \nu(S \times T)=m(S) \nu(T), \quad S \in \mathfrak{S}, \quad T \in \mathfrak{T} .
$$


The collection $\mathbb{S} \times \mathfrak{I}$ of all product sets $S \times T, S \in \mathbb{S}, T \in \mathfrak{T}$ is a semialgebra.

The systems $\left\langle\widetilde{S}_{\zeta} \times \mathfrak{T}\right\rangle_{\zeta \in Z}, \Lambda \times \nu, \Gamma \otimes \nu$ are formed by taking the products of individual members. The proofs of the following two lemmas follow directly from the definitions.

LemMA 3.1. If $\Lambda$ is $\Gamma$-compatible, then $\Lambda \times \nu$ is $\Gamma \otimes \nu$-compatible.

Lemma 3.2. If $\Lambda$ is $\Gamma$-closable, then $\Lambda \times \nu$ is $\Gamma \otimes \nu$-closable.

LemMa 3.3. Let $(\Sigma, \mathfrak{I}, \nu)$ be a finite measure space. Let $f: \Omega \times \Sigma \rightarrow \mathbb{R}$ be a $\Gamma \otimes \nu$-integrable function.

Then there exists a $\Gamma$-measurable set $\Omega_{0} \subseteq \Omega$ such that $\Omega \backslash \Omega_{0}$ is $\Gamma$-null, $f(\omega, \cdot)$ is $\nu$-integrable for all $\omega \in \Omega_{0}$, the function $\omega \rightarrow \int_{\Sigma} f(\omega, \sigma) d \nu(\sigma), \omega \in \Omega_{0}$ is $\Gamma$-integrable, and

$$
\int_{\Omega}\left[\int_{\Sigma} f(\omega, \sigma) d \nu(\sigma)\right] d \mu(\sigma)=\int_{\Omega \times \Sigma} f d \mu \otimes \nu
$$

for each $\mu \in \cup \Gamma$.

Proof. It is sufficient to consider the case where $f$ is non-negative. The function $f$ is $\Gamma \otimes \nu$-measurable, so it may be assumed that there exists an increasing sequence $s_{n}, n=1,2, \cdots$ of non-negative $\sigma(\widetilde{S}) \otimes \mathfrak{I}$-simple functions converging pointwise to $f$, by modifying $f \Gamma \otimes \nu$-a.e. if necessary. According to Fubini's theorem, the functions $g_{n}: \omega \rightarrow \int_{\Sigma} s_{n}(\omega, \sigma) d \nu(\sigma), \omega \in \Omega$, are $\mu$-integrable for each $\mu \in \cup \Gamma$, and $g_{n} \in L^{1}(\Gamma), n=1,2, \cdots$.

Now $g_{n}, n=1,2, \cdots$ is an increasing sequence in $L^{1}(\Gamma)$, and for each $\mu \in \cup \Gamma, \int_{\Omega} g_{n} d \mu \leq \int_{\Omega \times \Sigma} f d \mu \otimes \nu$, so by monotone convergence, the functions $g_{n}, n=1,2, \cdots$ converge in $L^{1}(\mu)$. The function $g=\lim _{n \rightarrow \infty} g_{n}$ is defined $\Gamma$-a.e.. Furthermore, it is measurable and belongs to $L^{1}(\Gamma)$.

The result follows on setting $\Omega_{0}$ equal to the set of all $\omega \in \Omega$ for which the sequence $g_{n}(\omega), n=1,2, \cdots$ converges.

The question now arises as to whether the other iterated integral exists, and is equal to the product integral. From the usual version of Fubini's theorem, it is clear that whenever there exists a $\nu$-measurable set $\Sigma_{0} \subseteq \Sigma$ such that $\Sigma \backslash \Sigma_{0}$ is $\nu$-null, and $f(\cdot, \sigma)$ is $\Gamma$-integrable, for all $\sigma \in \Sigma_{0}$, then the function $\sigma \rightarrow \int_{\Sigma} f(\omega, \sigma) d \mu(\omega), \sigma \in \Sigma_{0}$ is $\nu$-integrable, and 


$$
\int_{\Sigma}\left[\int_{\Omega} f(\omega, \sigma) d \mu(\omega)\right] d \nu(\sigma)=\int_{\Omega \times \Sigma} f d \mu \otimes \nu
$$

for each $\mu \in \cup \Gamma$.

However, such a set $\Sigma_{0}$ may not exist. An example of this phenomenon is given in [2] p. 170, where the family $\Gamma$ consists of the measures $|\langle m, \psi\rangle|, \psi \in X^{\prime},\|\psi\| \leq 1$ associated with the $X$-valued measure $m$.

Definition 3.4. The system $\Lambda$ is said to be countably $\Gamma$-closable if for each $m \in \cup \Lambda$ there exists a countable collection $E \subseteq \cup \Gamma$ such that $m\left(s_{n}\right) \rightarrow 0$ in $E$ whenever $s_{n}, n=1,2, \cdots$ are S-simple functions such that $s_{n} \rightarrow 0$ in $L^{1}(\Xi)$ and $m\left(s_{n}\right)$ converges in $E$ as $n \rightarrow \infty$.

Here, the family of seminorms consisting of the $L^{1}$-norm with respect to each measure in $E$ defines the topology of $L^{1}(E)$.

If $\Lambda$ is countably $\Gamma$-closable, then it is easy to see that it is $\Gamma$ closable. If this is the case, and $E$ is finite dimensional, then each set function $m \in \cup \Lambda$ is the restriction to $\widetilde{S}$ of a measure on $\sigma(\widetilde{S})$.

The properties of nuclear spaces referred to in the proof of the following theorem are given in [5].

TheOREM 3.5. Let $(\Sigma, \mathfrak{I}, \nu)$ be a finite measure space. Let $\Lambda$ be a $\Gamma$-closable family of E-valued set functions $m: \widetilde{S} \rightarrow E$. Let $f: \Omega \times \Gamma \rightarrow \mathbb{R}$ be a $\Gamma \otimes \nu-\Lambda \times \nu$-integrable function.

(i) Then there exists a $\Gamma$-measurable set $\Omega_{0} \subseteq \Omega$ such that $\Omega \backslash \Omega_{0}$ is $\Gamma$-null, $f(\omega, \cdot)$ is $\nu$-integrable, for all $\omega \in \Omega_{0}$, the function $\omega \rightarrow \int_{\Sigma} f(\omega, \sigma) d \nu(\omega)$, $\omega \in \Omega_{0}$ is $\Gamma$ - $\Lambda$-integrable, and

$$
\int_{\Omega}\left[\int_{\Sigma} f(\omega, \sigma) d \nu(\sigma)\right] d m(\omega)=\int_{\Omega \times \Sigma} f d m \times \nu
$$

for each $m \in \cup \Lambda$.

(ii) Suppose that $E$ is metrizable and nuclear, and $\Lambda$ is countably $\Gamma$-closable. If there exists a $\nu$-measurable set $\Sigma_{0} \subseteq \Sigma$ such that $\Sigma \backslash \Sigma_{0}$ is $\nu$-null and $f(\cdot, \sigma)$ is $\Gamma$-A-integrable for every $\sigma \in \Sigma_{0}$ then the function $\sigma \rightarrow$ $\int_{\Omega} f(\omega, \sigma) d m(\omega), \sigma \in \Sigma_{0}$ is v-integrable and

$$
\int_{\Sigma}\left[\int_{\Omega} f(\omega, \sigma) d m(\omega)\right] d \nu(\sigma)=\int_{\Omega \times \Sigma} f d m \times \nu
$$

for each $m \in \cup \Lambda$. 
Proof. Let $s_{\alpha}, \alpha \in A$ be a net of $\mathfrak{S} \times \mathfrak{I}$-simple functions converging in $L^{1}(\Gamma \otimes \nu, \Lambda \times \nu)$. By Lemma 3, there exists a $\Gamma$-measurable set $\Omega_{0} \subseteq \Omega$ such that $\Omega \backslash \Omega_{0}$ is $\Gamma$-null, $f(\omega, \cdot)$ is $\nu$-integrable, for all $\omega \in \Omega_{0}$, the function $\omega \rightarrow \int_{\Sigma} f(\omega, \sigma) d \nu(\sigma), \omega \in \Omega_{0}$ is $\Gamma$-integrable, and

$$
\int_{\Omega}\left[\int_{\Sigma} f(\omega, \sigma) d \nu(\sigma)\right] d \mu(\omega)=\int_{\Omega \times \Sigma} f d \mu \otimes \nu
$$

for each $\mu \in \cup \Gamma$. Moreover, $\lim _{\alpha \in A} \int_{\Sigma} s_{\alpha}(\cdot, \sigma) d \nu(\sigma)=\int_{\Sigma} f(\cdot, \sigma) d \nu(\sigma)$ in $L^{1}(\Gamma)$.

Since $s_{\alpha}, \alpha \in A$ converges in $L^{1}(\Gamma \otimes \nu, \Lambda \times \nu)$, for each $\xi \in W_{1}$ and $\zeta \in Z$,

$$
\int_{S} \int_{T} s_{\alpha}(\omega, \sigma) d \nu(\sigma) d m(\omega) \rightarrow \int_{S \times T} f d \nu \times m
$$

in $E$, uniformly for $m \in \Lambda_{\xi}, S \in \mathbb{S}_{\xi}$, and $T \in \mathfrak{T}$. The set function

$$
S \rightarrow \int_{S \times \Sigma} f d \nu \times m, S \in \mathbb{S}
$$

belongs to $\underset{\leftarrow}{b a_{\zeta \in Z}}\left(\widetilde{S}_{\zeta}, E\right)$, so $\int_{\Sigma} s_{\alpha}(\cdot, \sigma) d \nu(\sigma), \alpha \in A$ converges to $\int_{\Sigma} f(\cdot, \sigma) d \nu(\sigma)$ in the topology $\tau_{\Lambda}$. It follows that the function $\omega \rightarrow \int_{\Sigma} f(\omega, \sigma) d \nu(\sigma), \omega \in \Omega_{0}$ is $\Gamma$ - $\Lambda$-integrable, and

$$
\int_{\Omega}\left[\int_{\Sigma} f(\omega, \sigma) d \nu(\sigma)\right] d m(\omega)=\int_{\Omega \times \Sigma} f d m \times \nu
$$

for each $m \in \cup \Lambda$.

Suppose now that condition (ii) holds. For each $\xi \in W_{1}$ and $\zeta \in Z$,

$$
\int_{T} \int_{S} s_{\alpha}(\omega, \sigma) d m(\omega) d \nu(\sigma) \rightarrow \int_{S \times T} f d \nu \times m
$$

uniformly in $E$ for $m \in \Lambda_{\xi}, S \in \mathbb{S}_{\zeta}$ and $T \in \mathbb{I}$. The function $f(\cdot, \sigma)$ is $\Gamma$ - $\Lambda$-integrable, and in particular $\Gamma$-integrable, for every $\sigma \in \Sigma_{0}$. Fubini's theorem ensures that for every $S \in \sigma(\widetilde{S})$ and $T \in \mathfrak{I}$,

$$
\int_{S \times T} f d \mu \otimes \nu=\int_{T} \int_{S} f(\omega, \sigma) d \mu(\omega) d \nu(\sigma),
$$

so for each $\xi \in W_{0}, \int_{S} s_{\alpha}(\omega, \cdot) d \mu(\omega), \alpha \in A$ converges in $L^{1}(\nu)$ to $\int_{S} f(\omega, \cdot) d \mu(\omega)$ uniformly for $\mu \in \Gamma_{\xi}$ and $S \in \sigma(\widetilde{S})$.

Now choose $m \in \cup \Lambda$ and a countable family $\Xi \subseteq \cup \Gamma$ such that $r_{n} m(\Omega) \rightarrow 0$, whenever $r_{n} \rightarrow 0$ in $L^{1}(\Xi)$ and $r_{n} m(\Omega)$ converges in $E$ as $n \rightarrow \infty$, 
$r_{n} \in \operatorname{sim}(\Im), n=1,2, \cdots$.

We can find a (cofinal) subsequence $s_{n}, n=1,2, \cdots$ of $s_{\alpha}, \alpha \in A$ with the following properties; there exists a set $\Sigma_{1} \subseteq \Sigma_{0}$ of full $\nu$-measure such that for each $\mu \in \Xi$ and $\sigma \in \Sigma_{1}, \int_{S} s_{n}(\omega, \sigma) d \mu(\omega), n=1,2, \cdots$ converges to $\int_{S} f(\omega, \sigma) d \mu(\omega)$ uniformly for $S \in \sigma(\widetilde{S})$, and $\int_{\Omega} s_{n}(\omega, \sigma) d m(\omega)$ converges in $E$ for each $\sigma \in \Sigma_{1}$.

To see this, let $p_{j}(r)=\int_{\Sigma} \int_{\Omega}|r(\omega, \sigma)| d \mu_{j}(\omega) d \nu(\sigma), r \in L^{1}(\Gamma \otimes \nu), j=1,2, \ldots$ for $\Xi=\left\{\mu_{j}: j=1,2, \cdots\right\}$. The net $s_{\alpha}, \alpha \in A$ converges to $f$ in the seminorms $p_{j}, j=1,2, \cdots$ because it converges in $L^{1}(\Gamma \otimes \nu)$. Furthermore, the space $E$ is assumed to be metrizable, so for a given $\zeta \in Z$, there exists a subsequence $s_{n}^{\prime}, n=1,2, \cdots$ of $s_{\alpha}, \alpha \in A$ such that $p_{j}\left(f-s_{n}^{\prime}\right) \rightarrow 0$ as $n \rightarrow \infty$, for each $j=1,2, \cdots$ and $\int_{T} \int_{S} s_{n}^{\prime}(\omega, \sigma) d m(\omega) d \nu(\sigma), n=1,2, \cdots$ converges in $E$, uniformly for $T \in \mathfrak{T}$ and, $S \in \mathfrak{S}_{\zeta}$; if $\mathfrak{Q}$ is a countable collection of seminorms defining the topology of $E$, then for each $q \in \mathfrak{Q}$,

$$
\lim _{n, m \rightarrow \infty} \sup \left\{q\left(\int_{T} \int_{S}\left[s_{n}^{\prime}(\omega, \sigma)-s_{m}^{\prime}(\omega, \sigma)\right] d m(\omega) d \nu(\sigma)\right): T \in \mathfrak{I}, S \in \mathfrak{S}_{\zeta}\right\}=0 .
$$

Set $a_{j}=\sup \left\{p_{j}\left(s_{n}^{\prime}\right): n=1,2, \cdots\right\}, j=1,2, \ldots$ Then by dominated convergence $\lim _{n, m \rightarrow \infty} \sum_{j=1}^{\infty} \frac{p_{j}\left(s_{n}^{\prime}-s_{m}^{\prime}\right)}{\left(a_{j} 2^{j}\right)}=0$. Now choose a subsequence $s_{n}^{\prime \prime}$, $n=1,2, \cdots$ of $s_{n}^{\prime}, n=1,2, \cdots$ such that

$$
\begin{gathered}
\sup \left\{q\left(\int_{T} \int_{S}\left[s_{n+1}^{\prime \prime}(\omega, \sigma)-s_{n}^{\prime \prime}(\omega, \sigma)\right] d m(\omega) d \nu(\sigma)\right): T \in \mathfrak{T}, S \in \mathbb{S}_{\zeta}\right\} \rightarrow 0, \\
\sum_{j=1}^{\infty} \frac{p_{j}\left(s_{n+1}^{\prime \prime}-s_{n}^{\prime \prime}\right)}{\left(a_{j} 2^{j}\right)} \leq 2^{-n}
\end{gathered}
$$

for each $n=1,2, \cdots$ and $q \in \mathfrak{S}$. By the Beppo Levi convergence theorem, $\int_{S} s_{n}^{\prime \prime}(\omega, \sigma) d \mu(\omega), \quad n=1,2, \cdots$ converges to $\int_{S} f(\omega, \sigma) d \mu(\omega)$, uniformly for $S \in \mathbb{S}$, for $\nu$-almost all $\sigma \in \Sigma$.

Since $E$ is nuclear, the identity on $E$ is absolutely summing, so

$$
\sup \left\{\int_{\Sigma} q\left(\int_{S}\left[s_{n+1}^{\prime \prime}(\omega, \sigma)-s_{n}^{\prime \prime}(\omega, \sigma)\right] d m(\omega)\right) d \nu(\sigma): S \in \widetilde{S}_{\zeta}\right\} \rightarrow 0
$$

as $n \rightarrow \infty$, for each $q \in \mathfrak{\Omega}$.

By a diagonal procedure analogous to that above, there exists a set 
$\Sigma_{1}$ of full $\nu$-measure and a subsequence $s_{n}, n=1,2, \cdots$ of $s_{n}^{\prime \prime}, n=1,2, \cdots$ such that

$$
\begin{aligned}
& \lim _{n \rightarrow \infty} q\left(\int_{\Omega}\left[s_{n+1}(\omega, \sigma)-s_{n}(\omega, \sigma)\right] d m(\omega)\right)=0, \\
& \lim _{n \rightarrow \infty} \int_{\Omega}\left|s_{n}(\omega, \sigma)-f(\omega, \sigma)\right| d \mu(\omega)=0
\end{aligned}
$$

for each $q \in \mathfrak{\Omega}, \mu \in \Xi$ and $\sigma \in \Sigma_{1}$, and furthermore, $f(\cdot, \sigma)$ is $\Gamma$ - $\Lambda$-integrable for each $\sigma \in \Sigma_{1}$. In particular, for each $\sigma \in \Sigma_{1}$ there exist simple functions $r_{n}, n=1,2, \cdots$ such that

$$
\begin{aligned}
& \lim _{n \rightarrow \infty} q\left(\int_{\Omega} r_{n}(\omega, \sigma) d m(\omega)-\int_{\Omega} f(\omega, \sigma) d m(\omega)\right)=0, \\
& \lim _{n \rightarrow \infty} \int_{\Omega}\left|r_{n}(\omega, \sigma)-f(\omega, \sigma)\right| d \mu(\omega)=0
\end{aligned}
$$

for each $q \in \mathfrak{Q}, \mu \in \Xi$.

Since $\Lambda$ is countably $\Gamma$-closable, we must have

$$
\lim _{n \rightarrow \infty} q\left(\int_{\Omega} s_{n}(\omega, \sigma) d m(\omega)-\int_{\Omega} f(\omega, \sigma) d m(\omega)\right)=0
$$

for each $\sigma \in \Sigma_{1}$, otherwise we can extract a subsequence $t_{k}, k=1,2, \cdots$ from $r_{n}(\cdot, \sigma)-s_{m}(\cdot, \sigma), n, m=1,2, \cdots$ such that $t_{k} \rightarrow 0$ in $L^{1}(\Xi)$ and $m\left(t_{k}\right)$, $k=1,2, \cdots$ converges to an non-zero element of $E$.

It follows that the function $\sigma \rightarrow \int_{\Omega} f(\omega, \sigma) d m(\sigma), \sigma \in \Sigma_{0}$ is $\nu$-integrable and $\int_{\Sigma}\left[\int_{\Omega} f(\omega, \sigma) d m(\omega)\right] d \nu(\sigma)=\int_{\Omega \times \Sigma} f d m \times \nu$ for each $m \in \cup \Lambda$.

The following example shows that in Theorem 3.5 (ii), the existence of the integral with respect to the first variable of the function does not follow from the other conditions. The space $E$ is just $\mathbb{R}$, so it is surely metrizable and nuclear, $\Lambda$ and consists of the Lebesgue measure, trivially countably closable with respect to the family $\Gamma$ of restrictions of the Lebesgue measure to compact subsets of $] 0,1]$.

ExamLe 3.6. Let $f:] 0,1] \times[0,1] \rightarrow \mathbb{R}$ be the function defined by $f(x, t)=2^{n+1} \sin (n t) / n$ for $t \in[0,1]$ and $\left.\left.x \in\right] 2^{-n-1}, 2^{-n}\right], n=0,1,2, \cdots$.

The sequence $\{\sin (n \cdot) / n\}_{n=1}^{\infty}$ is summable in $L^{1}([0,1])$, so it is easy to check that $m(A \times B)=\int_{A} \int_{B} f(x, t) d t d x, A, B \in \mathfrak{B}([0,1])$ is separately $\sigma$ additive on $] 0,1] \times[0,1]$. 
Let $\lambda$ be the Lebesgue measure on $[0,1]$ and let $\Gamma$ be the family of restrictions of the Lebesgue measure to compact subsets of $] 0,1]$.

To see that the function $f$ is $\Gamma \otimes \lambda-\lambda \times \lambda$-integrable on $] 0,1] \times[0,1]$, we note that the sequence $\{\sin (n \cdot) / n\}_{n=1}^{\infty}$ is summable in $L^{1}([0,1])$, so there exist compact subsets $C_{n}$ of $\left.] 0,1\right]$ such that $\left.\left.\mid m(A \cap(] 0,1] \backslash C_{n}\right) \times B\right) \mid<1 / n$, for all $A, B \in \mathfrak{B}([0,1]), n=1,2, \cdots$. The semi-algebra $\subseteq$ is taken to be the Borel $\sigma$-algebra of $] 0,1]$, and the index set $Z=\{0\}$. Then because $f \chi_{C_{n} \times[0,1]} \rightarrow f$ in $L^{1}(\Gamma)$, and $f \chi_{C_{n} \times[0,1]} \lambda \otimes \lambda \rightarrow m$ uniformly on $\mathfrak{B}([0,1]) \times \mathfrak{B}([0,1])$ as $n \rightarrow \infty, f$ is $\Gamma \otimes \lambda$ - $\lambda \times \lambda$-integrable.

Now for every $t \in[0,1], \int_{0}^{1}|f(x, t)| d x=\sum_{n=1}^{\infty} \frac{|\sin (n t)|}{n}$, which diverges almost everywhere on $[0,1]$. A function on $] 0,1]$ is easily seen to be $\Gamma$ - $\lambda$-integrable if and only if it is $\lambda$-integrable, so for almost every $t \in[0,1]$, the function $x \rightarrow f(x, t), x \in] 0,1[$ is not $\Gamma$ - $\lambda$-integrable.

\section{§4. Application on Schrödinger semigroups}

Let $H_{0}=-\frac{1}{2} \Delta$ be the free Hamiltonian operator of a quantum system in $\mathbb{R}^{d}$. It was shown in [1], that a bounded potential $V: \mathbb{R}^{d} \rightarrow \mathbb{R}$ has the property that $\exp \left(-\mathfrak{i} \int_{0}^{t} V \circ X_{s}\right) d s$ is $M_{t}^{K}-M_{t}^{D}$-integrable for every $t>0$, and for every $z \in D, t>0$,

$$
\exp \left(-\mathfrak{i}\left(H_{0} / z+V\right) t\right)=\int_{\Omega} \exp \left(-\mathfrak{i} \int_{0}^{t} V \circ X_{s} d s\right) d M_{t}^{z}
$$

The proof there uses the Trotter product formula. In this section, the result is established by appealing to Fubini's theorem for closable set functions. The relationship between the operator $H_{0} / z+V$, and the generator of the semigroup $t \rightarrow \int_{\Omega} \exp \left(-\mathfrak{i} \int_{0}^{t} V \circ X_{s} d s\right) d M_{t}^{z}, t>0$ for unbounded $V$ is clarified as a by-product of the proof.

Let $\Gamma_{t}, \Lambda_{t}$ be the families of set functions associated with the free Schrödinger semigroup, defined in Section 2. If $\lambda$ is the Lebesgue measure on $\mathbb{R}$, then a $\Gamma_{t} \times \lambda-\Lambda_{t} \times \lambda$-integrable function on $\Omega \times[0, t]$ is said to be $M_{t}^{K} \otimes \lambda-M_{t}^{D} \times \lambda$-integrable.

THEOREM 4.1. Let $V: \mathbb{R}^{d} \rightarrow \mathbb{R}$ be a bounded Borel measurable function. Let $t>0$, and suppose that $f: \Omega \times[0, t] \rightarrow \mathbb{C}$ is the function defined by $f(\omega, s)=V(\omega(s)) \exp \left(-\mathfrak{i} \int_{0}^{s} V\left(\omega^{\prime}(r)\right) d r\right), \omega \in \Omega, s \in[0, t]$. 
Then $f$ is $M_{t}^{K} \otimes \lambda-M_{t}^{D} \times \lambda$-integrable on $\Omega \times[0, s]$ for all $s \in[0, t]$. Furthermore, the function $\omega \rightarrow \exp \left(-\mathfrak{i} \int_{0}^{s} V(\omega(r)) d r\right), \omega \in \Omega$ is $M_{t}^{K}-M_{t}^{D}$-integrable for all $s \in[0, t]$, and for all $z \in D$,

$$
R^{z}(s)=\int_{\Omega} \exp \left(-i \int_{0}^{s} V(\omega(r)) d r\right) d M_{s}(\omega)
$$

satisfies the following integral equation:

$$
R^{z}(s)=S^{z}(s)-\mathfrak{i} \int_{0}^{s} S^{z}(s-r) V R^{z}(r) d r, \quad s \in[0, t] .
$$

Proof. Let $O$ be the interior of the set $D$, The space $H(O)$ of functions analytic in $O$ with the topology of uniform convergence on compact subsets of $O$ is a nuclear Fréchet space [5] p. 107, so the space $H_{c}(D)$ of functions analytic in $O$, and continuous on $D$, with the topology of uniform convergence on compact subsets of $O$ is also nuclear and metrizable. The completion of $H_{c}(D)$ may be identified with $H(O)$.

It is a simple matter to check that for each $\varphi, \psi \in L^{2}\left(\mathbb{R}^{a}\right)$, the set function $\left(M_{t}^{o}, \phi, \psi\right)$ is countably $\Gamma_{t}$-closable; the measures $\left(M_{t}^{a}, \phi, \psi\right)$, $a=\left(1-n^{-1}\right) \mathfrak{i}, n=1,2, \cdots$ will do to verify the condition, on appealing to the Vitali convergence theorem. Similarly, $\left(M_{t}^{o} \times \lambda \phi, \psi\right)$ is countably $\Gamma_{t} \otimes \lambda$-closable.

The function $f: \Omega \times[0, t] \rightarrow \mathbb{C}$ is bounded and measurable, so it is surely $M_{t}^{K} \otimes \lambda$-integrable. Suppose that $V_{n}, n=1,2, \cdots$ are continuous functions converging almost everywhere to $V$ on $\mathbb{R}^{d}$, such that $\left\|V_{n}\right\|_{\infty} \leq$ $\|V\|_{\infty}, n=1,2, \ldots$. By choosing a subsequence, if necessary, it follows that $V_{n} \circ X_{s} \rightarrow V \circ X_{s}, s \in[0, t] M_{t}^{K} \otimes \lambda$-a.e.. Set

$$
f_{n, m}(\omega, s)=V_{n}(\omega(s)) \exp \left[-i \sum_{k=1}^{m} V_{n}\left(\omega\left(\frac{k s}{m}\right)\right) \frac{s}{m}\right] .
$$

Then $\lim _{n \rightarrow \infty} \lim _{m \rightarrow \infty} f_{n, m}=f, M_{t}^{K} \otimes \lambda$-a.e. on $\Omega \times[0, t]$. Each of the functions $f_{n, m}$ is $M_{t}^{K} \otimes \lambda-M_{t}^{D} \times \lambda$-integrable, and for each $S \in \mathbb{S}_{t} \times \mathfrak{B}[0,1]$, the norms of $f_{n, m} M_{t}^{z} \otimes \lambda(S), n, m=1,2, \cdots, z \in O$ are uniformly bounded, so the convergence is uniform as $z$ ranges over compact subsets of $O$ by the Vitali convergence theorem. Dominated convergence, and the Vitali convergence theorem shows that $f_{n, m}$ converges to $f$ in $L^{1}\left(M_{t}^{K} \otimes \lambda, M_{t}^{o} \times \lambda\right)$.

Now for each $\omega \in \Omega, f(\omega, s)=\mathfrak{i} d / d s \exp \left(-\mathfrak{i} \int_{0}^{s} V(\omega(r)) d r\right), s \in[0, t]$, so Theorem 3.5 shows that $\omega \rightarrow \exp \left(-\mathfrak{i} \int_{0}^{s} V(\omega(r)) d r\right)$, is $M_{t}^{K}-M_{t}^{D}$-integrable 
and

$$
\begin{aligned}
\frac{1}{\dot{i}} \int_{\Omega \times[0, t]} f d M_{t}^{z} \times \lambda & =\int_{\Omega} \int_{0}^{t} d / d s \exp \left(-\mathfrak{i} \int_{0}^{s} V(\omega(r)) d r\right) d s d M_{t}^{z} \\
& =R^{z}(t)-S^{z}(t) .
\end{aligned}
$$

For each $s \in[0, t]$, a similar argument shows that $f(\cdot, s)$ is $M_{t}^{K}-M_{t}^{o}$ integrable and furthermore,

$$
\int_{\Omega} f(\omega, s) d M_{t}^{z}(\omega)=S^{z}(t-s) V R^{z}(s), \quad z \in O
$$

Therefore Theorem 3.5 shows that

$$
\int_{\Omega \times[0, t]} f d M_{t}^{z} \times \lambda=\mathfrak{i} R^{z}(t)-\mathfrak{i} S^{z}(t)=\int_{0}^{t} S^{z}(t-s) V R^{z}(s) d s
$$

for every $z \in O$. It remains to establish that for each $\varphi, \psi \in L^{2}\left(\mathbb{R}^{d}\right)$, and $S \in \mathfrak{S}_{t} \times \mathfrak{B}[0,1]$, the function $z \rightarrow \int_{S} f d M_{t}^{z} \times \lambda, z \in O$ is the restriction to $O$ of a continuous function on $D$.

The solution $R^{z}(t)=\int_{\Omega} \exp \left(-\mathfrak{i} \int_{0}^{t} V(\omega(r)) d r\right) d M_{t}^{z}(\omega)$ of the operator equation above is obtained by taking the Laplace transform of both sides and integrating by parts, as follows.

For each $\varphi \in L^{2}\left(\mathbb{R}^{d}\right), \psi \in \mathfrak{D}\left(H_{0}\right)$,

$$
\partial / \partial t\left(S^{z}(t-s) V R^{z}(s) \varphi, \psi\right)=-i / z\left(S^{z}(t-s) V R^{z}(s) \varphi, H_{0} \psi\right)
$$

exists for all $s>0$, and it is continuous for $0 \leq s \leq t$, therefore

$$
\begin{aligned}
d / d t \int_{0}^{t}\left(S^{z}(t-s) V R^{z}(s) \varphi, \psi\right) d s= & -i / z \int_{0}^{t}\left(S^{z}(t-s) V R^{z}(s) \varphi, H_{0} \psi\right) d s \\
& +\left(V R^{z}(t) \varphi, \psi\right), \quad t \geqslant 0 .
\end{aligned}
$$

By taking the Laplace transform of both sides of equation (1), integrating by parts, and substituting in (1) again, we obtain the equation

$$
\begin{aligned}
& \lambda\left(U^{z}(\lambda) \varphi, \psi\right)+\mathfrak{i} / z\left(U^{z}(\lambda), H_{0} \psi\right)+\mathfrak{i}\left(U^{z}(\lambda) \varphi, V \psi\right) \\
& \quad=\lambda\left(\left(\lambda+\mathfrak{i} / z H_{0}\right)^{-1} \varphi, \psi\right)+\mathfrak{i} / z\left(\left(\lambda+\mathfrak{i} / z H_{0}\right)^{-1} \varphi, H_{0} \psi\right)=(\varphi, \psi),
\end{aligned}
$$

where $U^{z}(\lambda)=\int_{0}^{\infty} e^{-\lambda t} R^{z}(t) d t, \lambda>0$. It follows that

$$
\lambda+\mathfrak{i}\left(H_{0} / \bar{z}+V\right)^{*}=\lambda+\mathfrak{i}\left(H_{0} / z+V\right)
$$

maps the range of $U^{z}(\lambda)$ onto $L^{2}\left(\mathbb{R}^{d}\right)$ injectively for every $z \in O$, so 
$U^{z}(\lambda)=\left[\lambda+\mathfrak{i}\left(H_{0} / z+V\right)\right]^{-1}$. The essential uniqueness of inverse Laplace transforms shows that $R^{z}(t)=\exp \left(-\mathfrak{i}\left(H_{0} / z+V\right), t\right), t>0$.

The result follows from dominated convergence, by noting that for each $s \in[0, t], z \rightarrow \exp \left(-\mathrm{i}\left(H_{0} / z+V\right) s\right), z \in D$ is continuous for the strong operator topology, by strong resolvent convergence.

CoRollaRy 4.2. Let $V: \mathbb{R}^{d} \rightarrow \mathbb{R}$ be a bounded Borel measurable function. Then for every $t>0$,

$$
\exp \left(-\mathfrak{i}\left(H_{0}+V\right) t\right)=\int_{\Omega} \exp \left(-\mathfrak{i} \int_{0}^{t}(V(\omega(r)) d r) d M_{t}^{1}(\omega)\right.
$$

In the following corollary, $L_{\mathrm{loc}}^{2}\left(\mathbb{R}^{d}\right)$ denotes the space of real valued functions whose square is integrable over any compact set in $\mathbb{R}^{d}$.

CoRollary 4.3. Suppose that $V \in L_{\mathrm{loc}}^{2}\left(\mathbb{R}^{d}\right)$, and the function $\omega \rightarrow$ $\exp \left(-\mathfrak{i} \int_{0}^{t} V(\omega(r)) d r\right), \omega \in \Omega$ is $M_{t}^{K}-M_{t}^{D}$-integrable for every $t>0$.

For every $z \in D, t>0$, let $T_{z}$ be the adjoint $\left(H_{0} / \bar{z}+V\right)^{*}$ of $\left(H_{0} / \bar{z}+V\right)$ : $\mathfrak{D}\left(H_{0}\right) \cap \mathfrak{D}(V) \rightarrow L^{2}\left(\mathbb{R}^{d}\right)$ restricted to the range of $U^{z}(\lambda)=\int_{0}^{\infty} e^{-\lambda t} R^{z}(t) d t$. Then $-i T_{z}$ is the generator of a continuous contraction semigroup on $L^{2}(\mathbb{R})$, and for each $t>0$.

$$
\exp \left(-\mathfrak{i} T_{z} t\right)=\int_{\Omega} \exp \left(-\mathfrak{i} \int_{0}^{t} V \circ X_{s} d s\right) d M_{t}^{z} .
$$

Proof. For any $V$ as above, set

$$
R^{z^{\prime}}(V, t)=\int_{\Omega} \exp \left(-i \int_{0}^{t} V(\omega(r)) d r\right) d M_{t}^{z}(\omega), \quad U^{z}(V, \lambda)=\int_{0}^{\infty} e^{-\lambda t} R^{z}(t) d t
$$

Then there exist bounded measurable functions $V_{n m}, n, m=1,2, \cdots$ converging to $V$ on $\mathfrak{S}(V)$ as $m \rightarrow \infty$ and $n \rightarrow \infty$, and for each $t>0$, the operators $R^{z}\left(V_{n m}, t\right)$ converge to $R^{z}(V, t)$ in the strong operator topology, uniformly for $z$ in compact subsets of $D^{\circ}$. The convergence for $z$ on the positive imaginary axis follows by dominated convergence, and because $\left\|R^{z}\left(V_{n m}, t\right)\right\| \leq 1, n, m=1,2, \cdots, z \in D^{\circ}$, the convergence for $z$ in the interior $D^{\circ}$ of $D$ is a consequence of the Vitali convergence theorem.

Now for $n, m=1,2, \cdots$, and $z \in D^{\circ}, \varphi \in L^{2}\left(\mathbb{R}^{d}\right), \psi \in \mathfrak{S}\left(H_{0}\right) \cap \mathfrak{D}(V)$,

$$
\lambda\left(U^{z}\left(V_{n m}, \lambda\right) \varphi, \psi\right)+\mathfrak{i} / z\left(U^{z}\left(V_{n m}, \lambda\right) \varphi, H_{0} \psi\right)+\mathfrak{i}\left(U^{z}\left(V_{n m}, \lambda\right) \varphi, V_{n m} \psi\right)=(\varphi, \psi),
$$

by (2), so by dominated convergence. 


$$
\lambda\left(U^{z}(V, \lambda) \varphi, \psi\right)+\mathfrak{i} / z\left(U^{z}(V, \lambda) \varphi, H_{0} \psi\right)+\mathfrak{i}\left(U^{z}(V, \lambda) \varphi, V \psi\right)=(\varphi, \psi) .
$$

Because $\exp \left(-i \int_{0}^{t} V \circ X_{r} d r\right)$ is $M_{t}^{K}-M_{t}^{D}$-integrable, $z \rightarrow R^{z}(V, t), z \in D$ is continuous in the weak operator topology, so by dominated convergence again, $z \rightarrow U^{z}(V, \lambda), z \in D$ is also continuous in the weak operator topology. Therefore, for all $z \in D$,

$$
\left(\left[\lambda+\dot{i}\left(H_{0} / \bar{z}+V\right)^{*}\right] U^{z}(V, \lambda) \varphi, \psi\right)=(\varphi, \psi) .
$$

Consequently, $U^{z}(V, \lambda)=\left(\lambda+\mathfrak{i} T_{z}\right)^{-1}$, so by the Hille-Yosida theory, $-i T_{z}$ is the generator of $R^{z}(V, t), t \geq 0$, proving the claim.

In the case that the functions $\omega \rightarrow \exp \left( \pm i \int_{0}^{s} V(\omega(r)) d r\right), \omega \in \Omega$ are $M_{t}^{K}-M_{t}^{D}$-integrable for all $s \in[0, t], t>0$, we can see precisely when $H_{0}+V$ has a self-adjoint extension equal to the generator of the semigroup $R^{1}$. Suppose that $V \in L_{\mathrm{loc}}^{2}\left(\mathbb{R}^{d}\right)$ as above.

For $z \in D$, let $R_{ \pm}^{z}(s)=\int_{\Omega} \exp \left( \pm i \int_{0}^{s} V(\omega(r)) d r\right) d M_{s}^{z}(\omega)$, for all $s>0$, and set $U_{ \pm}^{z}(\lambda)=\int_{0}^{\infty} e^{-\lambda t} R_{ \pm}^{z}(t) d t$, for every $\lambda>0$. The range of the operator $U_{ \pm}^{z}(1)$ (which is the same as the range of $U_{ \pm}^{z}(\lambda), \lambda>0$ ) is denoted by $\Re_{ \pm}^{z}$. If $\Re_{-}^{1}=\Re_{+}^{-1}$, then the generator of the semigroup $R^{1}$ is a self-adjoint extension of $H_{0}+V$ with domain $\Re_{-}^{1}$. If, in addition, $\left(H_{0}+V\right)^{*}$ is selfadjoint on $\Re_{-}^{1}$, then $H_{0}+V$ is essentially self-adjoint. The operator $H_{0}+V$ is self-adjoint when $\mathfrak{R}_{-}^{1}=\Re_{+}^{-1}=\mathfrak{D}\left(H_{0}\right) \cap \mathfrak{D}(V)$.

The various cases are illustrated by the potentials $V(x)=-\frac{\beta}{|x|^{2}}$, $x \in \mathbb{R}^{5}, \beta>0$, in $\mathbb{R}^{5}$ where $\mathfrak{D}(V) \supset \mathfrak{D}\left(H_{0}\right)[6]$. 


\section{REFERENCES}

[1] B. Jefferies, Integration with respect to closable set functions, J. Funct. Anal., 66 (1986), 381-405.

[2] I. Kluvánek, Operator valued measures and perturbations of semigroups, Arch. Rat. Mech. Anal., 81 (1983), 161-180.

[ 3 ] I. Kluvánek, Integration and the Feynman-Kac formula Studia Mathematica, 86 (1987), 35-57.

[4] I. Kluvánek and J. Knowles, Vector Measures and Control Systems, North Holland, Amsterdam, 1976.

[ 5 ] H. H. Schaefer, Topological Vector Spaces, Springer-Verlag, New York, Heidelberg, Berlin 1971.

[6] B. Simon, Essential self-adjointness of Schrödinger operators with positive potentials, Math. Ann., 201 (1973), 211-220.

Department of Mathematics

The University of Wollongong

P.O. Box 1144 Wollongong

N.S.W. 2500

Australia

Present Address:

School of Mathematics, Physics

Electronics and Computing

Macquarie University

N.S.W. 2109

Australia 